NASA Technical Memorandum 106077

AIAA-93-2177

\title{
Compound Curvature Laser Window Development
}

Vincent G. Verhoff

Lewis Research Center

Cleveland, Ohio

Prepared for the

29th Joint Propulsion Conference and Exhibit

cosponsored by the AIAA, SAE, ASME, and ASEE

Monterey, California, June 28-30, 1993 


\title{
COMPOUND CURVATURE LASER WINDOW DEVELOPMENT
}

\author{
Vincent G. Verhoff \\ National Aeronautics and Space Administration \\ Lewis Research Center \\ Cleveland, Ohio 44135
}

Abstract

The NASA Lewis Research Center has developed and implemented a unique process for forming flawless compound curvature laser windows. These windows represent a major part of specialized, nonintrusive laser data acquisition systems used in a variety of compressor and turbine research test facilities. This report summarizes the main aspects of compound curvature laser window development. It is an overview of the methodology and the peculiarities associated with the formulation of these windows. Included in this discussion is new information regarding procedures for compound curvature laser window development.

\section{Introduction}

An increased interest in fundamental research in turbines and compressors has created a need for nonintrusive optical flow measurement systems. The stateof-the-art systems used in obtaining detailed velocity data are called nonintrusive laser data acquisition systems. These systems seed the airflow with small particles that flow through a fringe pattern created by intersecting laser beams. Data are obtained by measuring the pulsating light reflected as the seed particles pass through the fringe pattern.

Optically clear laser windows are used for the laser beams and reflected light to pass through. Normally the laser window glass used in wind tunnels is approximately 1.0-in.-thick flat quartz. For turbine and compressor testing facilities, however, the windows are approximately 0.100 in. thick and have compound curvatures. The difference between the two windows is shown in Fig. 1.

Since laser windows are molded to the flow surface profile of the turbine and compressor casings, their size and curvature cannot be altered. Window thickness, on the other hand, can vary. Thinner windows are desirable because they minimize error, but the glass must maintain high strength with reduced thickness. Reference 1 provides a detailed explanation of compound curvature laser window development. This paper summarizes the results from Ref. 1 and provides some new information regarding the development process.
Background

Laser systems are commonly used at NASA Lewis Research Center. Most of these systems are located in the engine component test facilities that test compressor and turbine rotors and components. The windows are usually located over rotating hardware where typical instrumentation cannot be used.

Overall safety is of primary concern. To uphold safety practices, the laser windows are hydrostatically pressure tested to $\mathbf{1 . 5}$ times the maximum operating pressures of the facilities. Operating pressures for various facilities range from 1.3 psia to 72 psia. Laser windows are also thermally qualified at facility temperatures and pressures if thermal differentials are significant. The laser windows are tested on both concave and convex surfaces to uphold the safety requirements.

\section{Glass Selection}

Several types of glass have been used for laser window development at NASA Lewis. Sodium-lime, borosilicate, and sodium-alumino-silicate glass are among these types. On the basis of the Corning glass code $0317,{ }^{2}$ sodium-alumino-silicate glass is the preferred glass for laser windows where high strength is needed. This high strength is achieved through chemical strengthening, which is not possible with other glass types. This increased strength from chemical strengthening allows the use of thinner laser windows, which reduce spatial error and produce higher quality laser data. At near ambient temperature and pressure operating conditions, high-strength windows are generally not required and any of the glass types could be suitable. Sodium-lime windows are the least costly alternative.

An additional benefit of the sodium-aluminosilicate laser window is that a failure will cause the window to shatter in tiny particles, only millimeters in cross-sectional area. This characteristic is advantageous since the smaller particles contain less kinetic energy and are less likely to damage blades or rotors. The particles from other failed glass windows are relatively large in cross-sectional area. Figure 2 shows a comparison of failed strengthened and unstrengthened laser window glass. 
To help select the correct type of laser window glass for the application intended, an analytical equation is being developed. This equation includes the parameters of glass load area, thickness, working pressure, and bond/contact area.

\section{Mold Design}

The mold material that offers the best results for forming laser windows is machinable ceramic because it produces the best accuracies. Machinable ceramic can be machined to closer tolerances and surface finishes than graphite. Reference 1 stated that graphite was the best suitable material; however, further development indicates that machinable ceramic offers the best results.

The molds for laser window development consist of a male and female mold, as shown in Fig. 3. The male mold is machined to match the internal flow path surfaces, whereas the female mold is machined to these coordinates plus the glass thickness. The overall dimensions of the molds are 1 in. greater than the actual size of the laser window glass. Both molds have threaded holes on the perimeter to fit alignment bars.

To maintain surface quality, accurate machining and polishing of the molds used for laser window development are essential. The molds are machined and polished to a tolerance of $0.003 \mathrm{in}$. of the desired contour. After finish machining, these molds are polished to within an $8 \mathrm{Ra}$ surface finish.

All slumping components are machined out of same material to ensure similar coefficients of thermal expansion. Orientation of the slumping component configuration is shown in Fig. 4.

\section{Glass Molding}

Inert gas furnaces are preferred for slumping because of the heating characteristics of the molds. Other types of furnaces usually introduce contaminants into the slumping environment that degrade glass surface quality.

Cleanliness of the molds, glass, and furnace is critical to glass quality. These components should be thoroughly cleaned at the beginning of every slumping operation.

The forming temperature of laser windows is found by an iterative process. The mean annealing temperature of about $1100^{\circ} \mathrm{F}$ is the theoretical starting temperature for new laser window development. Adequate visual inspection of the window after slumping will indicate whether the temperature should be increased or decreased. The ideal slumping temperature for compoundcurvature laser window development is usually within 2 percent of the annealing temperature. The procedure for laser window development is detailed in Ref. 1.

Visual inspection of the slumped laser window will provide adequate information to alter the slumping temperature. If the slumping temperature is excessive, surface imperfections will be apparent. These surface imperfections will appear along the plane of severe compound curvature or inflection point. This problem can be solved by decreasing the slumping temperature by 1 to 2 percent of the annealing temperature.

Laser window development can be a lengthy process, but once the parameters are found for a particular laser window, reproduction is routine. The last process in the formulation of laser windows is to anneal the glass. This relieves residual stresses developed in the glass during the development process. Glass annealing also helps achieve glass strength consistencies from one laser window to another.

\section{Glass Edging}

Previous methods of edging the laser windows to size involved scoring the glass to the desired size, then breaking the glass along this scored edge. During this process, small fragments of glass are broken away along the line of scoring, creating voids in the glass. Unfortunately, minute cracks along the edge of the glass remain after the glass is broken (Fig. 5). These cracks weaken the glass and cause failure of the glass under load.

Minimizing cracks is important during the edging process. A perfected process for edging glass using a numerically controlled water-jet cutting machine has been developed and is recommended for cutting laser windows. The machine abrades glass away along the desired cut line, reducing glass fragmentation. The water-jet cut edge of the glass is less densely populated with cracks, and crack penetration is less severe when compared with scored glass.

The desired laser window dimensions are programmed into the numerically controlled water-jet cutting machine. The laser window glass is then secured into position by pressing it into clay and the entire assembly is then submersed under water to further assist in absorbing the high cutting frequencies of the water jet.

After the laser window glass is cut to size, the edges are rounded. The minimum radius of the rounded 
edges is equivalent to half the glass thickness, as shown in Fig. 6.

\section{Glass Strengthening}

Sodium-alumino silicate laser windows are chemically strengthened through ion exchange to endure facility operating pressures and temperatures, increase their relative impact strength, and minimize glass fracture particle size. These windows are chemically strengthened to contain a 0.010 -in. or greater compression layer. Other types of glass do not benefit from the chemical strengthening process. Detailed information on the chemical strengthening process is given in Refs. 3 to 8 .

\section{Glass Bonding to Frame}

The laser window frames are specially designed to pocket and hold the glass. Figure 7 shows this unique design. The laser window glass is bonded to the window frame using a high-strength semiflexible adhesive. The laser window glass is actually floated in the laser window frame with 0.030 - to 0.050 -in. bonding medium between the laser window glass and all laser window frame surfaces. Figure 8 shows a cross section of the laser window frame, glass, and bonding medium. The bonding strength is increased drastically by a semicircular groove located in the lower and inner edge of the laser window frame.

Accurate positioning of laser window glass into the frame is a crucial step in obtaining quality laser data. Laser window glass can be positioned precisely by using a specifically designed gluing fixture to hold the alignment of the laser window glass with respect to the laser window frame. Gluing fixtures are machined to the identical contour and dimensions of the male molds.

Figure 9 shows the assembly stackup for bonding the laser window glass to the frame. The gluing fixture has an inscribed inner channel 0.125 in. in depth which matches the circumference of the laser window glass. Without this escape channel, bonding medium would get trapped over the frame or glass, resulting in a nonuniform transition between the laser window glass and frame. Duct seal is used to hold the alignment of the laser window glass to the gluing fixture.

Figure 10 represents a cross section of the bonding process. The laser window glass is aligned onto the gluing fixture by positioning the outer perimeter of the laser window glass with the inner edge of the bonding medium escape channel. The bonding medium is then applied to the supporting edge of the laser window frame. The frame is aligned on the gluing fixture using dowel pins.

\section{Window Proof-Testing Procedure}

At NASA Lewis, the turbine and compressor casings into which the laser windows are located are evaluated in accordance with boiler vessel codes to ensure their structural integrity and safety. The normal process for evaluating facility hardware is hydrostatic testing. To uphold the structural integrity and safety of these facilities, the laser windows are also hydrostatically tested after assembly. A detailed procedure for prooftesting is found in Ref. 1 .

\section{Concluding Remarks}

High-quality contoured windows are a key part of laser anemometry systems for compressor and turbine facilities. The present development process ensures accurate tolerances and flawless quality of these laser windows. Although they are tested for flaws, laser windows should be considered and treated as a fragile material. Routine visual inspection and hydrostatic evaluations are considered important to their integrity.

Future experiments indicate the need for larger laser windows to increase the fields of unobstructed view. Future windows must also endure higher pressures and temperature gradients. With the present development processes, these laser windows should successfully withstand these future requirements.

\section{$\underline{\text { References }}$}

1. Verhoff, V.G., "Three-Dimensional Laser Window Formation." NASA RP-1280, 1992.

2. Corning Glass Code 0317 Specification Sheet, Corning, Inc., Corning, NY, 1990.

3. McLellan, I., and Shgnd, G.W. II, Glass Engineering Handbook, Third ed., McGraw Hill, 1984.

4. Nordberg, M.E., Moehel, M.E., Gartinke, H.M., and Olcott, J.S., "Strengthening by Ion Exchange," American Ceramic Society Journal, Vol. 47, No. 5, May 1964, pp. 215-219.

5. Hagy, H.E., "Design Strength of a Chemically Strengthened Glass," Central Glass Ceramic Research Institute Bulletin, Vol 13, No. 1, 1966, pp. 29-31. 
6. Blizard, J.R., "Chemically Strengthened Glass," SAE Paper 680485, 1968.

7. Miska, H.A., "Understanding the Basics of Chemically Strengthened Glass," Materials Engineering, Vol. 83, No. 6, 1976, pp. 38-40.
8. Olcott, J.S., "Strengthening of Glass," Science, Vol. 140, No. 3572, June 14, 1963, pp. 1189-1193.

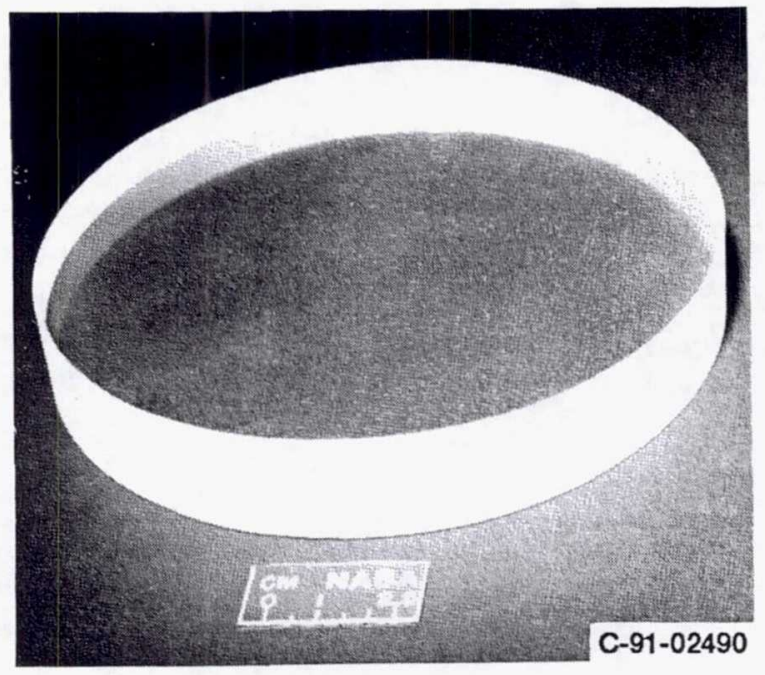

(a) Wind tunnel window.

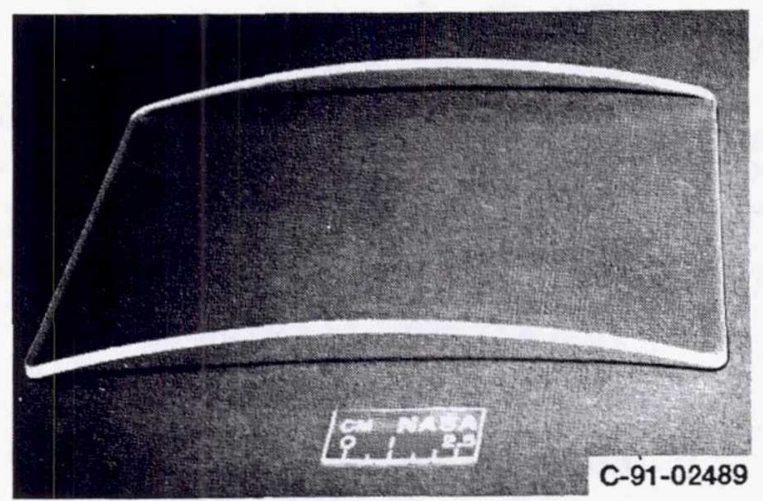

(b) Turbine/compressor window.

Figure 1.-Typical laser windows. 


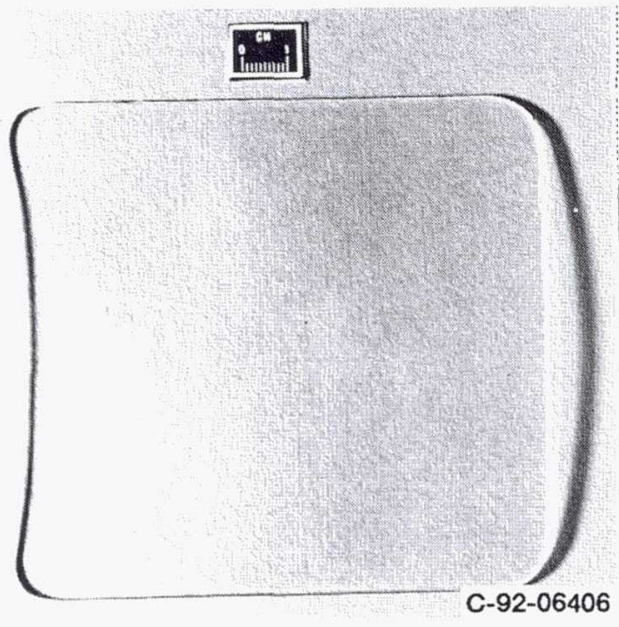

(a) Laser window before failure.

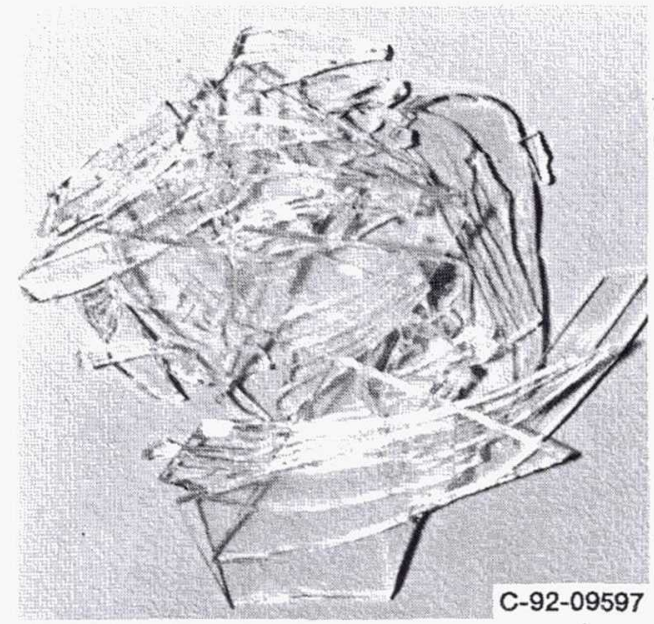

(b) Unstrengthened laser window after failure.

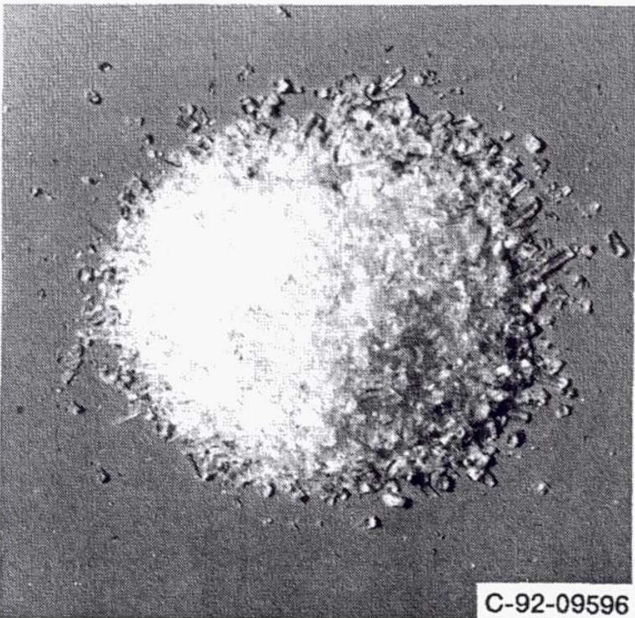

(c) Strengthened laser window after failure.

Figure 2.-Comparison of failed strengthened and unstrengthened laser window glass.

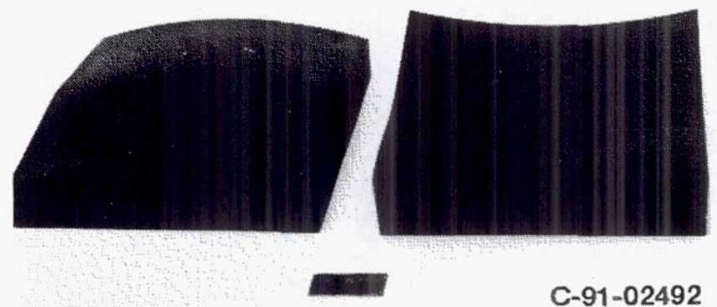

Figure 3.-Laser window male and female mold.

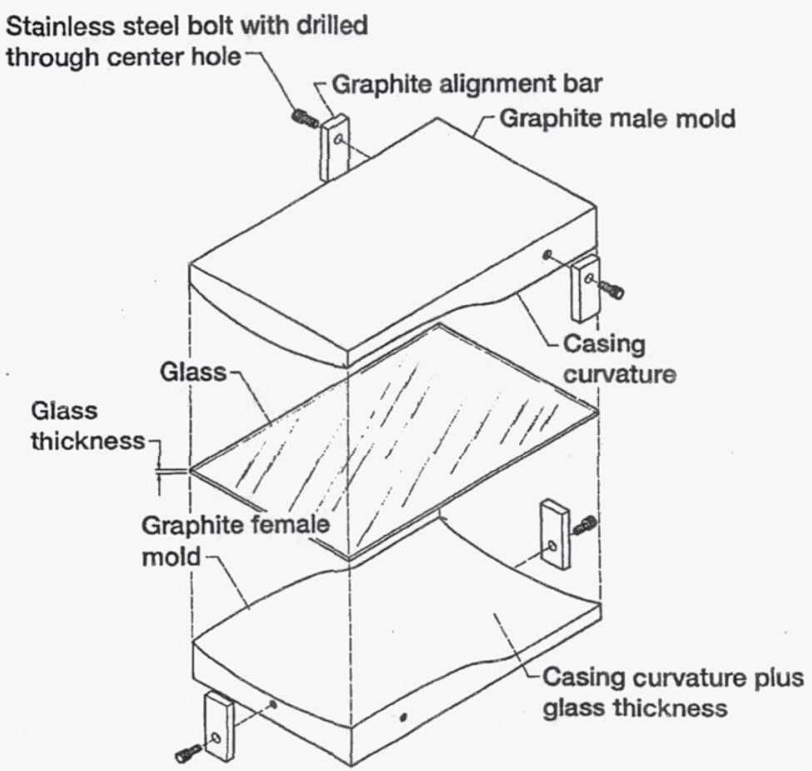

Figure 4.-Slumping components configuration.

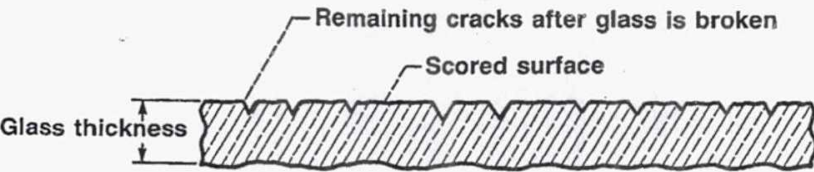

Figure 5.-Edged window glass by scoring. 


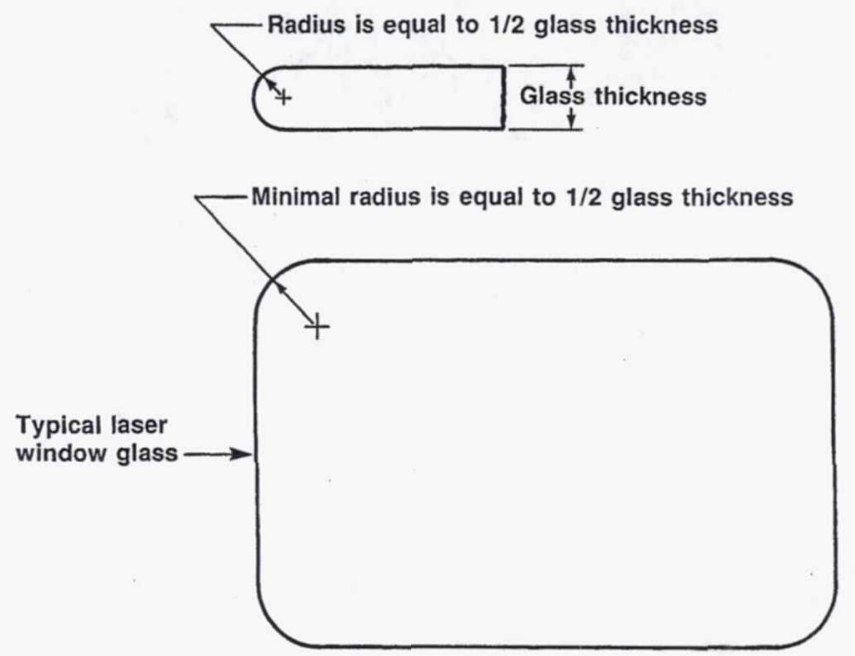

Figure 6.-Rounded edges of typical laser window.

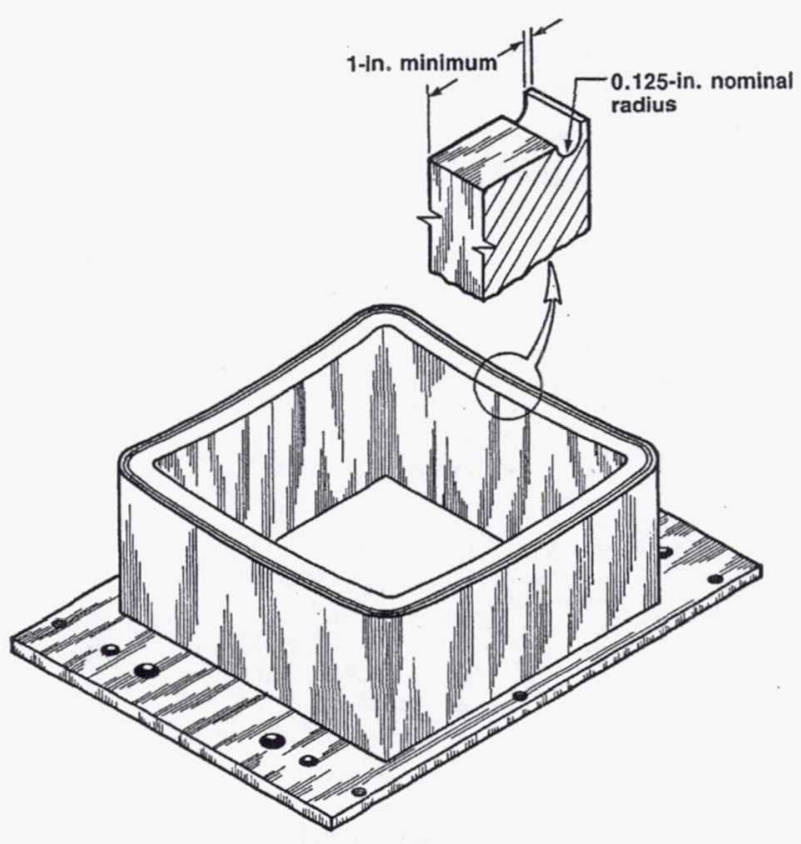

Figure 7.-Typical laser window frame.

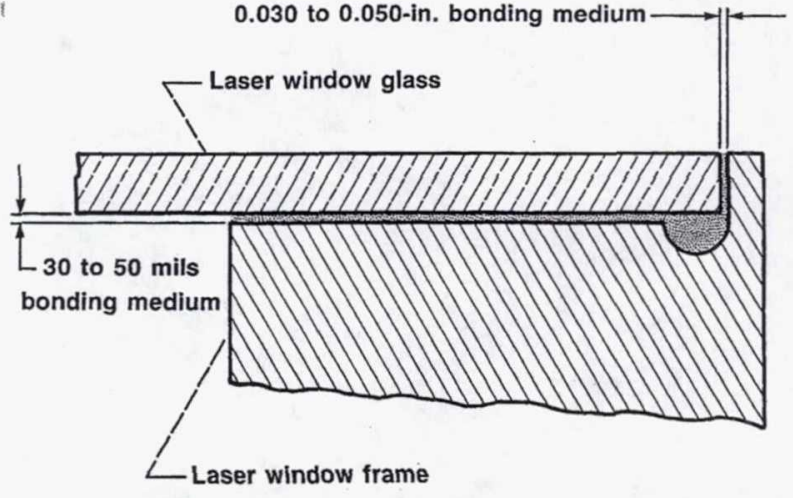

Figure 8.-Cross section of laser window frame, glass, and bonding medium. 


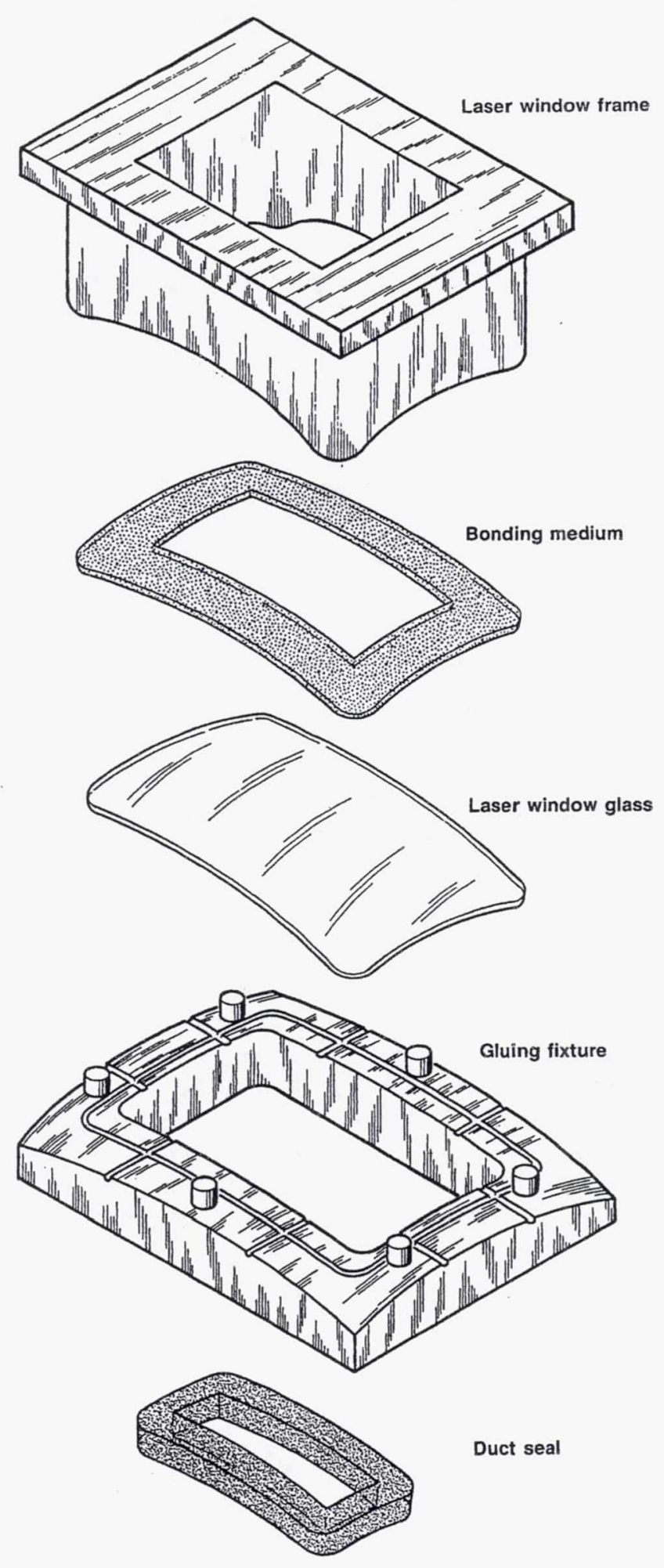

Figure 9.-Assembly stackup of laser window bonding process.

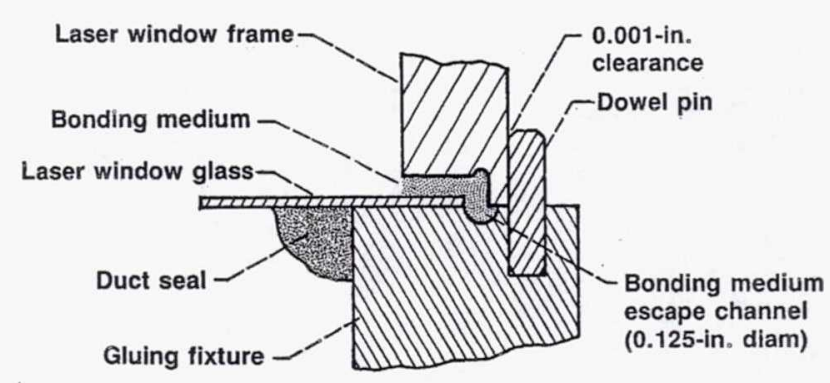

Figure 10.-Cross section of laser window bonding process. 


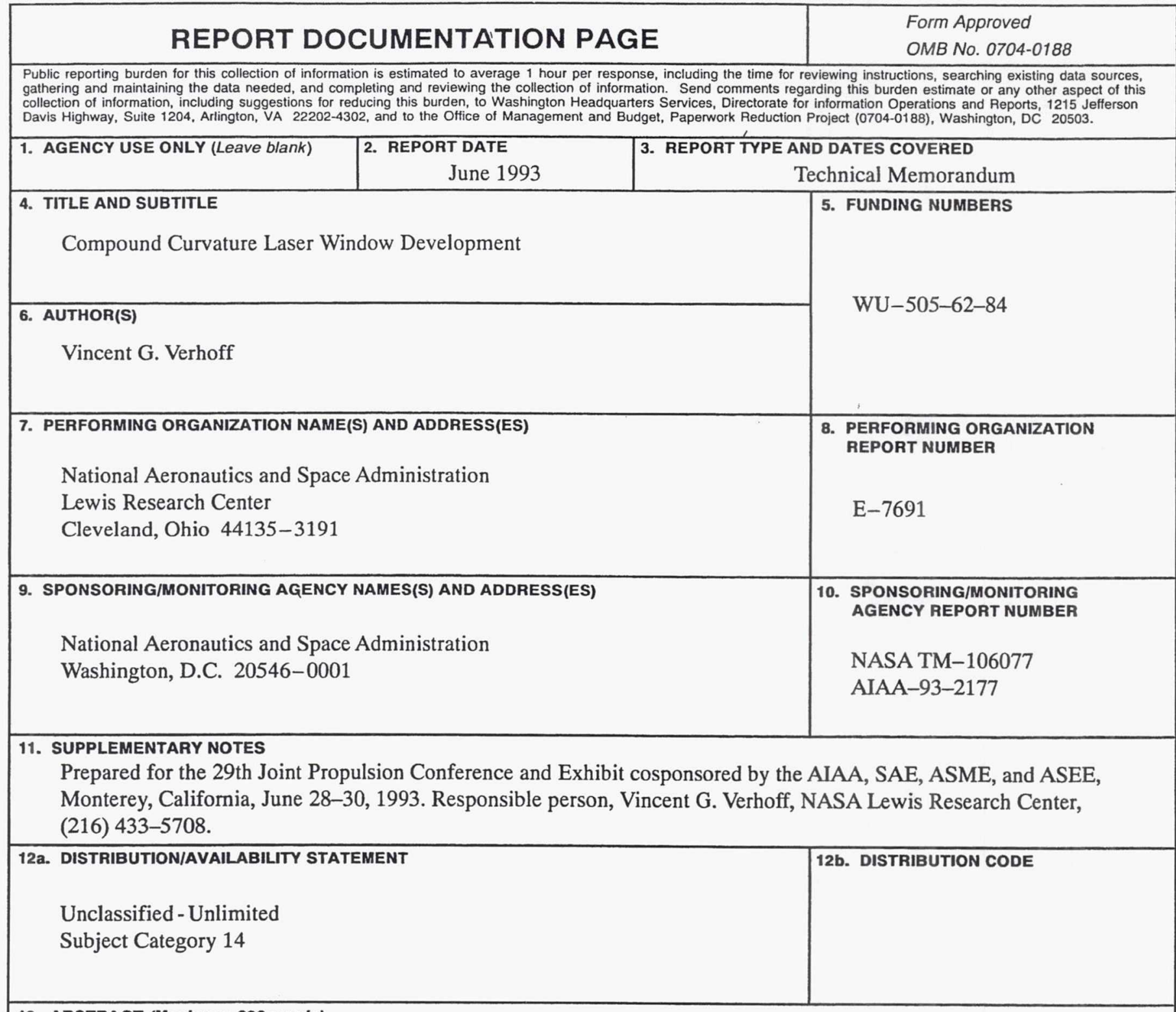

\section{ABSTRACT (Maximum 200 words)}

The NASA Lewis Research Center has developed and implemented a unique process for forming flawless compound curvature laser windows. These windows represent a major part of specialized, nonintrusive laser data acquisition systems used in a variety of compressor and turbine research test facilities. This report summarizes the main aspects of compound curvature laser window development. It is an overview of the methodology and the peculiarities associated with the formulation of these windows. Included in this discussion is new information regarding procedures for compound curvature laser window development.

14. SUBJECT TERMS
Windows; Laser windows; Three-dimensional glass; Curved glass; Slumped glass; Molded glass; Chemical strengthened glass

17. SECURITY CLASSIFICATION OF REPORT

Unclassified
18. SECURITY CLASSIFICATION OF THIS PAGE Unclassified
19. SECURITY CLASSIFICATION OF ABSTRACT Unclassified 